\title{
Ground-state energy of an exciton-(LO)-phonon system in two dimensions
}

\author{
B. Gerlach \\ Institut für Physik, Universität Dortmund, D-44221 Dortmund, Germany \\ F. Luczak \\ Departement Natuurkunde, Universiteit Antwerpen, Universiteitsplein 1, B-2610 Wilrijk, Belgium
}

(Received 30 April 1997; revised manuscript received 27 August 1997)

\begin{abstract}
This paper presents a variational study of the ground-state energy of an exciton-(LO)-phonon system in two spatial dimensions. It complements a preceding publication, providing a general ( $D$-dimensional) outline and an explicit treatment of the case $D=3$. The exciton-phonon interaction is of the Fröhlich type. Making use of functional-integral and variational techniques, we obtain upper bounds on the ground-state energy. The present study-like the previous one-has two major intentions: First, we demonstrate for the problem under consideration that one can profitably use a nonharmonic trial action within the functional-integral framework. Second, we show that our lowest bound is an analytical function of the electron-phonon coupling parameter, being completely smooth throughout the whole parameter region. This is in contrast to earlier conjectures, and excludes phenomena such as self-trapping or self-shrinking, but is consistent with rigorous qualitative results for the true ground-state energy. We include a critical comparison of our results with previous ones.
\end{abstract} [S0163-1829(98)06203-1]

\section{INTRODUCTION AND STATEMENT OF THE PROBLEM}

All considerations to follow are based on Fröhlich's Hamiltonian, generalized for the interaction of an electron and a hole with a (LO) phonon branch (see, e.g., Haken ${ }^{1}$ ):

$$
\begin{aligned}
H:= & \sum_{n=1}^{2} \frac{p_{n}^{2}}{2 m_{n}}-\frac{\vec{e}^{2}}{\epsilon_{\infty}\left|\vec{q}_{1}-\vec{q}_{2}\right|}+\sum_{\vec{k}} \hbar \omega a_{\vec{k}}^{+} a_{\vec{k}} \\
& +\sum_{n=1}^{2}(-1)^{n} \sum_{\vec{k}}\left\{\frac{g_{\vec{k}}}{\sqrt{V}} e^{i \vec{k} q_{n}} a_{\vec{k}}+\text { H.c. }\right\} .
\end{aligned}
$$

$n=1$ and 2 refers to electron and hole, $\vec{p}_{n}, \vec{q}_{n}$, and $m_{n}$ are the corresponding momentum and position operators and band masses, and $\epsilon_{0}$ and $\epsilon_{\infty}$ the low- and high-frequency limits of the dielectric function. We included the permittivity of the free space (which is usually abbreviated as $\epsilon_{0}$ too) into our definition of the charge, $\bar{e}^{2}:=e^{2} / 4 \pi \epsilon_{0}, e$ being the hole charge. Furthermore, $a_{\vec{k}}$ and $a_{\vec{k}}^{+}$are the annihilation and creation operators for phonons with wave vector $\vec{k}$ and dispersion $\omega_{\vec{k}} \equiv \omega$, and $V$ is the (two-dimensional) quantization volume. Finally, the coupling $g_{\vec{k}}$ is connected with $\vec{k}$ as follows:

$$
g_{\vec{k}}:=\frac{g}{\sqrt{k}}, \quad g:=-i \sqrt{\pi \bar{e}^{2} \hbar \omega\left(\frac{1}{\epsilon_{\infty}}-\frac{1}{\epsilon_{0}}\right)} .
$$

We recall that the particle-phonon coupling strength can be characterized by the dimensionless parameter

$$
\alpha_{m}:=\frac{\vec{e}}{2 \hbar \omega} \sqrt{\frac{2 m \omega}{\hbar}}\left(\frac{1}{\epsilon_{\infty}}-\frac{1}{\epsilon_{0}}\right),
$$

$m$ being the particle mass.
Combining functional-integral and variational methods, we demonstrated in a previous paper ${ }^{2}$ that the following inequality is true:

$$
\begin{aligned}
E_{0} \leqslant & \widetilde{E}_{0}+\left\langle\widetilde{\phi}_{0}\left|U_{c}-U\right| \widetilde{\phi}_{0}\right\rangle+\frac{\hbar \omega}{\pi} \int_{0}^{\infty} d x\left\{\ln \left(1+\frac{h(x)}{x^{2}}\right)\right. \\
& \left.-\frac{h(x)}{x^{2}+h(x)}\right\}-\int d^{2} k\left\{\frac{\left|g_{\vec{k}}\right|^{2}}{(2 \pi)^{2}} \int_{0}^{\infty} d u \exp \{-\hbar \omega u\right. \\
& \left.-\frac{\hbar^{2} k^{2}}{M \hbar \omega \pi} P(\hbar \omega u)\right\} \oiint_{\mu} e^{u\left(\widetilde{E}_{0}-\widetilde{E}_{\mu}\right)} \\
& \left.\times\left|\sum_{n}(-1)^{n}\left\langle\widetilde{\phi}_{0}\left|e^{i \vec{k} \gamma_{n} \vec{q}}\right| \widetilde{\phi}_{\mu}\right\rangle\right|^{2}\right\} .
\end{aligned}
$$

Here $E_{0}$ is the ground-state energy of the system of interest, and $U_{c}$ the Coulomb potential between electron and hole. $\mu$ and $M$ are the familiar abbreviations for the reduced and total mass of the exciton. $\widetilde{H}:=\left(p^{2} / 2 \mu\right)+U(\vec{r})$ denotes a two-dimensional trial Hamiltonian with eigenfunctions $\widetilde{\phi}_{\mu}$ and eigenvalues $\widetilde{E}_{\mu}$. This Hamiltonian mimics the relative motion of the electron and hole. We assume that $\widetilde{H}$ has an isolated ground state $\widetilde{\phi}_{0}$. The function $h(x)$, in turn, models the center-of-mass motion. $h(x)$-just like the operator $\widetilde{H}$-is at our disposal, and may be chosen such as to lower the quoted bound on $E_{0}$ as far as possible. Finally, $P(y)$ is connected with $h(x)$ as follows:

$$
P(y):=\int_{0}^{\infty} d x \frac{1-\cos (x y)}{x^{2}+h(x)} .
$$

We stress that the above bound on $E_{0}$ is flexible enough to account for the interesting limits of the polaronic and bare 
exciton, as we demonstrated earlier (see Ref. 3). The reader will notice that the treatment of the center-of-mass motion is polaronlike. It is guaranteed that the strong-coupling limit of the exciton is described with the same accuracy as in Feynman's treatment of the polaron; the relative deviation from the exact result is less than $2 \%$. It would be tempting to find the absolute minimum of the bound as a functional of $U$ and $h$. Unfortunately, the corresponding conditions turn out to be nonlinear integrodifferential equations of high complexity. Therefore, we made special variational choices, which are motivated by former results from polaron and exciton theory (again, we refer to our previous paper; ${ }^{2}$ all above remarks concerning the limiting behavior remain valid):

$$
h(x):=\frac{\left(v^{2}-w^{2}\right) x^{2}}{w^{2}+x^{2}}, \quad U(\vec{r}):=-\frac{\lambda \vec{e}^{2}}{\boldsymbol{\epsilon}_{\infty} r} .
$$

Here $v, w(v \geqslant w)$, and $\lambda \geqslant 0$ are variational parameters. Inserting Eq. (6) into Eq. (4), one arrives at

$$
\begin{aligned}
E_{0} \leqslant & 4 R_{\infty}\left(\lambda^{2}-2 \lambda\right)+\frac{\hbar \omega}{2} \frac{(v-w)^{2}}{v} \\
& -\sum_{m=0}^{\infty} \frac{1}{m !}\left(\frac{\hbar^{2}}{2 M \hbar \omega} \frac{v^{2}-w^{2}}{v^{3}}\right)^{m} \int d^{2} k\left\{\frac{\left|g_{k}\right|^{2}}{(2 \pi)^{2}} k^{2 m}\right. \\
& \times \exp \left\{-\frac{\hbar^{2} k^{2}}{2 M \hbar \omega} \frac{v^{2}-w^{2}}{v^{3}}\right\} \sum_{n, n^{\prime}}(-1)^{n+n^{\prime}} \\
& \left.\times\left\langle\widetilde{\phi}_{0}\left|e^{i \vec{k} \gamma_{n} \vec{q}}(\widetilde{H}-z)^{-1} e^{-i \vec{k} \gamma_{n^{\prime}} \vec{q}}\right| \widetilde{\phi}_{0}\right\rangle\right\},
\end{aligned}
$$

where we introduced the Rydberg unit of energy:

$$
R_{\infty}:=\frac{\mu \bar{e}^{4}}{2 \hbar^{2} \epsilon_{\infty}^{2}}, \quad z:=-4 R_{\infty} \lambda^{2}-\hbar \omega(1+m v)-\frac{w^{2}}{v^{2}} \frac{\hbar^{2} k^{2}}{2 M}
$$

In the remainder of this paper we shall be concerned with a numerical evaluation of the above inequality. It is interesting to notice that a whole class of bounds can be derived from the present one by truncation of the $m$ sum in expression (7). To prove this, one may insert the Laplace transform of the resolvent $(\widetilde{H}-z)^{-1}$, and will find that every $m$ contribution is positive on its own. Of course, all of these bounds are weaker than the present one. We anticipate the numerical result that an $m$-truncation may generate a nonanalytical bound. In any case we need an explicit representation of the resolvent $(\widetilde{H}-z)^{-1}$ to proceed. We briefly comment on this point in Sec. II.

\section{AN EXPLICIT EXPRESSION FOR THE RESOLVENT OF THE HYDROGEN HAMILTONIAN IN TWO DIMENSIONS}

It is well known that $\left\langle\vec{r}\left|(\widetilde{H}-z)^{-1}\right| \vec{r}^{\prime}\right\rangle$ can explicitly be calculated in the case of a hydrogen Hamiltonian, the spatial dimension $D$ being arbitrary (see the work of Hostler ${ }^{4}$ ). For $D=2$, one finds, in Hostler's paper,

$$
\left.\langle\vec{r}|(\widetilde{H}-z)^{-1}\right)\left|\vec{r}^{\prime}\right\rangle=\frac{1}{2 \pi} \sum_{s=-\infty}^{\infty} e^{i s\left(\varphi-\varphi^{\prime}\right)} g_{s}\left(r, r^{\prime}, z\right),
$$

where

$$
\begin{aligned}
g_{s}\left(r, r^{\prime}, z\right)= & \frac{\mu \kappa a}{\hbar^{2}} \frac{\Gamma\left(\frac{1}{2}+|s|-\kappa\right)}{\Gamma(1+2|s|)} \\
& \times \frac{W_{\kappa,|s|}\left(\frac{2 r_{>}}{\kappa a}\right) M_{\kappa,|s|}\left(\frac{2 r_{<}}{\kappa a}\right)}{\sqrt{r r^{\prime}}} .
\end{aligned}
$$

In these equations, $r$ and $\varphi$ denote the length and the azimuthal angle of the two-dimensional vector $\vec{r}$, and $r_{>}$and $r_{<}$ the maximum and minimum of $r$ and $r^{\prime} . W_{\kappa,|s|}$ and $M_{\kappa,|s|}$ are Whittaker functions as defined, e.g., in the book by Erdelyi et al. ${ }^{5}$ Finally, we introduced the abbreviations

$$
a:=\frac{\hbar^{2} \epsilon_{\infty}}{\mu \lambda \bar{e}^{2}}=\frac{a_{B}}{\lambda}, \quad \kappa:=\sqrt{\frac{R_{\infty} \lambda^{2}}{|z|}}<\frac{1}{2},
$$

$a_{B}$ being the familiar Bohr radius. It proves important to reformulate Eq. (10) by means of an integral representation due to Buchholz, ${ }^{6}$ which reads as follows:

$$
\begin{aligned}
W_{\kappa,|s|}(z) M_{\kappa,|s|}\left(z^{\prime}\right)= & \frac{\sqrt{z z^{\prime}}}{\Gamma\left(\frac{1}{2}+|s|-\kappa\right)} \Gamma(1+2|s|) \\
& \times \int_{0}^{\infty} d \alpha e^{-\left[\left(z+z^{\prime}\right) / 2\right] \cosh \alpha} \\
& \times \operatorname{coth}^{2 \kappa}\left(\frac{\alpha}{2}\right) I_{2|s|}\left(\sqrt{z z^{\prime}} \sinh \alpha\right),
\end{aligned}
$$

$$
0 \leqslant z^{\prime}<z
$$

Here $I_{2|s|}(z)$ is a modified Bessel function as defined in Ref. 5. Utilizing Buchholz's formula in Eq. (10), we arrive at

$$
\begin{aligned}
g_{s}\left(r, r^{\prime}, z\right)= & \frac{2 \mu}{\hbar^{2}} \int_{0}^{\infty} d \alpha e^{-\left[\left(r+r^{\prime}\right) / \kappa a\right] \cosh \alpha} \operatorname{coth}^{2 \kappa}\left(\frac{\alpha}{2}\right) \\
& \times I_{2|s|}\left(\frac{2 \sqrt{r r^{\prime}}}{\kappa a} \sinh \alpha\right) .
\end{aligned}
$$

Turning back to Eq. (9), we may insert this result for $g_{s}\left(r, r^{\prime}, z\right)$ to find an expression for the resolvent which is appropriate for a numerical evaluation of bound (7) on $E_{0}$.

\section{UPPER BOUNDS ON THE GROUND-STATE ENERGY}

The central quantity to be inserted into expression (7) for the bound of interest is 


$$
\begin{aligned}
\frac{32 \mu a^{2}}{\hbar^{2}} F_{m}(k):= & \sum_{n, n^{\prime}}(-1)^{n+n^{\prime}} \\
& \times\left\langle\widetilde{\phi}_{0}\left|e^{i \vec{k} \gamma_{n} \vec{q}}(\widetilde{H}-z)^{-1} e^{-i \vec{k} \gamma_{n^{\prime}} \vec{q}}\right| \widetilde{\phi}_{0}\right\rangle \\
= & \sum_{n, n^{\prime}}(-1)^{n+n^{\prime}} \iint d^{2} r d^{2} r^{\prime} e^{i \vec{k}\left(\gamma_{n} \vec{r}-\gamma_{n^{\prime}} \vec{r}^{\prime}\right)} \\
& \times\left\langle\vec{r}\left|(\widetilde{H}-z)^{-1}\right| \vec{r}^{\prime}\right\rangle \widetilde{\phi}_{0}^{*}(\vec{r}) \widetilde{\phi}_{0}\left(\vec{r}^{\prime}\right)
\end{aligned}
$$

Recalling Eqs. (9) and (13) for the resolvent, and using the familiar exponential for the ground-state wave function $\widetilde{\phi}_{0}(\vec{r})$, we can perform the $\vec{r}$ and $\vec{r}^{\prime}$ integrations as well as the $s$ summation. As in the three-dimensional case, the calculation is lengthy but elementary. Therefore, we restrict ourselves to a precise description of the steps to be done: First, angular integrations yield Bessel functions with index $s$. One is left with two radial integrations, which are Laplace transforms of products of Bessel functions. Having performed one of these (see Ref. 7), one should evaluate the $s$-summation (see Ref. 8), before the last integration (a Laplace transform of a Bessel function with index zero) is done. Finally, one arrives at

$$
\begin{aligned}
F_{m}(k)= & \sum_{n, n^{\prime}} \int_{0}^{\infty} d \alpha \operatorname{coth}^{2 \kappa}\left(\frac{\alpha}{2}\right)\left\{\delta_{n, n^{\prime}} \frac{\chi^{2}+\zeta^{2}}{I_{n}^{3}}-\left(1-\delta_{n, n^{\prime}}\right)\right. \\
& \left.\times\left(\frac{\chi^{2}+\zeta^{2}}{J^{3 / 2}}-\frac{3 \zeta^{2} \chi^{2} k^{2} a^{2}}{J^{5 / 2}}\right)\right\},
\end{aligned}
$$

where we introduced the abbreviations

$$
\begin{gathered}
\chi:=2+\frac{\cosh \alpha}{\kappa}, \quad \zeta:=\frac{\sinh \alpha}{\kappa}, \\
I_{n}:=\left(k a \gamma_{n}\right)^{2}+\chi^{2}-\zeta^{2}, \\
J:=\left(\chi^{2}-k^{2} a^{2}\left|\gamma_{1} \gamma_{2}\right|-\zeta^{2}\right)^{2}+\chi^{2} k^{2} a^{2} .
\end{gathered}
$$

It is useful to perform a final substitution in expression (15): we denote

$$
y:=\tanh ^{2}\left(\frac{\alpha}{2}\right),
$$

$$
\begin{aligned}
F_{m}(k)= & \sum_{n, n^{\prime}} \int_{0}^{1} d y y^{-\kappa-(1 / 2)} \\
& \times\left(\delta_{n n^{\prime}} \frac{\left(2+\frac{1}{\kappa}\right)^{2}+2\left(\frac{3}{\kappa^{2}}-4\right) y+\left(\frac{1}{\kappa}-2\right)^{2} y^{2}}{\bar{I}_{n}^{3}}\right. \\
& -\left(1-\delta_{n n^{\prime}}\right) \\
& \times\left(\frac{\left(2+\frac{1}{\kappa}\right)^{2}+2\left(\frac{3}{\kappa^{2}}-4\right) y+\left(\frac{1}{\kappa}-2\right)^{2} y^{2}}{\bar{J}^{3 / 2}}\right. \\
& \left.\left.-\frac{\left.12 k^{2} a^{2} y\left[\frac{1}{\kappa}+2+\left(\frac{1}{\kappa}-2\right) y\right]^{2}\right\}}{\kappa^{2} \bar{J}^{5 / 2}}\right\}\right)
\end{aligned}
$$

In Eq. (19), we defined

$$
\begin{aligned}
\bar{I}_{n} & :=\left(2+\frac{1}{\kappa}\right)^{2}+k^{2} a^{2} \gamma_{n}^{2}-\left[\left(\frac{1}{\kappa}-2\right)^{2}+k^{2} a^{2} \gamma_{n}^{2}\right] y, \\
\bar{J}:= & \left\{\left(2+\frac{1}{\kappa}\right)^{2}-k^{2} a^{2}\left|\gamma_{1} \gamma_{2}\right|-\left[\left(\frac{1}{\kappa}-2\right)^{2}\right.\right. \\
& \left.\left.-k^{2} a^{2}\left|\gamma_{1} \gamma_{2}\right|\right] y\right\}^{2}+k^{2} a^{2}\left[\left(2+\frac{1}{\kappa}\right)+\left(\frac{1}{\kappa}-2\right) y\right]^{2} .
\end{aligned}
$$

Returning to inequality (7) for the ground-state energy, we insert expressions (19) for $F_{m}(k)$ and (2) for $g$, leading us to the final result

$$
\begin{aligned}
\frac{E_{0}}{R_{0}} \leqslant & \frac{1}{(1-\xi)^{2}}\left\{4\left(\lambda^{2}-2 \lambda\right)+\frac{(v-w)^{2}}{2 v \eta^{2}}\right. \\
& -\frac{16\left(\sigma+\sigma^{-1}\right) \xi}{\eta^{3} \lambda^{2}} \sum_{m=0}^{\infty} \frac{1}{m !}\left(\frac{v^{2}-w^{2}}{v^{3}}\right)^{m} \\
& \left.\times \int_{0}^{\infty} d x x^{2 m} e^{-\left[\left(v^{2}-w^{2}\right) / v^{3}\right] x^{2}} F_{m}\left(\sqrt{\frac{2 M \omega}{\hbar}} x\right)\right\} \\
= & : B(v, w, \lambda) .
\end{aligned}
$$

The reader will notice that we used the static Rydberg unit

$$
R_{0}:=\frac{\mu \bar{e}^{4}}{2 \hbar^{2} \epsilon_{0}^{2}}
$$

as the energy scale. In doing so, we followed the convention of many papers, which are concerned with the threedimensional case; it is convenient to allow for a direct comparison of two- and three-dimensional results. Furthermore, we introduced the three dimensionless material parameters

and arrive at 
TABLE I. Results for the ground-state energy bounds $B_{0}(v, v, 1)$, and $\overline{B_{0}}$ for specified parameter values $\eta^{2}=\frac{1}{4}$ and $\xi$ $=0.5$.

\begin{tabular}{ccc}
\hline \hline$\sigma^{2}$ & $B_{0}(v, v, 1)$ & $\overline{B_{0}}$ \\
\hline 0.010 & -71.788 & -74.108 \\
0.020 & -53.967 & -55.996 \\
0.050 & -38.742 & -40.295 \\
0.100 & -31.625 & -32.804 \\
0.200 & -27.156 & -28.022 \\
0.500 & -24.242 & -24.758 \\
1.000 & -23.512 & -24.073 \\
\hline \hline
\end{tabular}

$$
\eta^{2}:=\frac{R_{\infty}}{\hbar \omega}, \quad \sigma^{2}:=\frac{m_{1}}{m_{2}}, \quad \xi:=1-\frac{\epsilon_{\infty}}{\epsilon_{0}} .
$$

One can easily derive from Eqs. (24) and (11) that

$$
\frac{1}{\kappa^{2}}=4+\frac{1}{\eta^{2} \lambda^{2}}\left(1+m v+\frac{w^{2}}{v^{2}} x^{2}\right)
$$

is true. Consequently, the bound $B(v, w, \lambda)$ is entirely defined by $\eta, \sigma, \xi$ alone. The remaining task is to minimize $B(v, w, \lambda)$ as a function of the variational parameters $v, w$, and $\lambda$.

\section{NUMERICAL RESULTS AND COMPARISON WITH PREVIOUS WORK}

The minimization of $B(v, w, \lambda)$ with respect to $v, w$, and $\lambda$ requires a numerical treatment. We proceed in exactly the same way as in our former paper on $D=3$. To achieve a compact presentation of our results, it will prove useful to introduce again the notation $B_{k}(v, w, \lambda)$ for a truncated bound: $B_{k}(v, w, \lambda)$ is derived from $B(v, w, \lambda)$ by omission of all terms in the $m$ sum, having an $m$ value larger than $k$. We remind the reader that $B_{k}(v, w, \lambda)$ is in fact a true upper bound on $E_{0} / R_{0}$, and decreases monotoneously with increasing $k$.

We begin the discussion by analyzing a restricted class of bounds. It is related to the analyticity problem concerning the ground-state energy as function of $\alpha_{M}$ : let us consider the case $v=w$. Recalling the derivation (see the general part in Ref. 2), one realizes that the variational action assumes a free center-of-mass motion if $v=w$. In this case, we find

$$
B(v, v, \lambda)=B_{0}(v, v, \lambda)
$$

the right-hand side being independent of $v$. If we additionally put $\lambda=1$, the trial action is precisely that of an uncoupled exciton-phonon system. Consequently, $B_{0}(v, v, 1)$ will produce the result of second-order perturbation theory for $E_{0} / R_{0}$. One can easily improve the bound by calculating

$$
\overline{B_{0}}:=\inf _{\lambda} B_{0}(v, v, \lambda) \text {. }
$$

Tables I-III contain a collection of results for $B_{0}(v, v, 1)$ as well as $\overline{B_{0}} \cdot B_{0}$ will be explained below.

In comparison with the three-dimensional case, we find no qualitative difference. However, phonon-induced effects
TABLE II. Results for the ground-state energy bounds $B_{0}(v, v, 1), \overline{B_{0}}$, and $B_{0}$ for specified parameter values $\eta^{2}=1$ and $\xi=0.5$.

\begin{tabular}{cccc}
\hline \hline$\sigma^{2}$ & $B_{0}(v, v, 1)$ & $\overline{B_{0}}$ & $B_{0}$ \\
\hline 0.010 & -39.157 & -40.359 & -48.387 \\
0.020 & -30.838 & -31.679 & $-v=w-$ \\
0.050 & -24.095 & -24.533 & \\
0.100 & -21.164 & -21.407 & \\
0.200 & -19.444 & -19.579 & \\
0.500 & -18.361 & -18.436 & \\
1.000 & -18.144 & -18.209 & $-v=w-$ \\
\hline \hline
\end{tabular}

are more pronounced for $D=2$. In particular, the onset of the strong-coupling regime is shifted to smaller values of the relevant parameter,

$$
\alpha_{M}=\frac{\overline{e^{2}}}{2 \hbar \omega} \sqrt{\frac{2 M \omega}{\hbar}}\left(\frac{1}{\epsilon_{\infty}}-\frac{1}{\epsilon_{0}}\right)=\eta \xi\left(\sigma+\frac{1}{\sigma}\right) .
$$

This is in complete analogy to the results of free-polaron theory. Xiaoguang, Peeters, and Devreese ${ }^{9}$ demonstrated within the Feynman approach that the two-dimensional ground-state energy for a given value of $\alpha$ can be related to the corresponding three-dimensional energy, if $\alpha$ is replaced by $3 \pi \alpha / 4$.

We now turn to the more general bounds

$$
B_{k}:=\inf _{v, w, \lambda} B_{k}(v, w, \lambda) .
$$

These admit an infimum for $v \neq w$, which is equivalent to a nonfree center-of-mass motion. In any case, $B_{k} \leqslant \overline{B_{0}}$, and in particular $B_{0} \leqslant \overline{B_{0}}$, is guaranteed. Comparing $B_{0}$ with $\overline{B_{0}}$, we discover the same phenomenon as in the three-dimensional case. If $\eta$ and $\xi$ are fixed, there may exist a critical value of $\alpha_{M}$ (or, equivalently, $\sigma$ ) in the following sense: If $\alpha_{M}$ $<\alpha_{M}^{c}, B_{0}=\overline{B_{0}}$ is true, $v$ and $w$ being equal. If $\alpha_{M}>\alpha_{M}^{c}$, $B_{0}<\overline{B_{0}}$ will be found, the minimizing values of $v$ and $w$ being not equal. For $\alpha_{M}=\alpha_{M}^{c}, B_{0}$ is a nonanalytical function of $\alpha_{M}$. Interestingly enough, this nonanalytical behavior of a variational bound has been discovered in many different approaches-primarily in the three-dimensional case, but easily transferable to two dimensions. Moreover, there exist phenomenological arguments, which also (seem to) indicate

TABLE III. Results for the ground-state energy bounds $B_{0}(v, v, 1), \overline{B_{0}}$, and $B_{0}$ for specified parameter values $\eta^{2}=4$ and $\xi=0.5$.

\begin{tabular}{cccc}
\hline \hline$\sigma^{2}$ & $B_{0}(v, v, 1)$ & $\overline{B_{0}}$ & $B_{0}$ \\
\hline 0.010 & -24.555 & -24.942 & -45.440 \\
0.020 & -21.087 & -21.281 & -27.017 \\
0.050 & -18.513 & -18.579 & $-v=w-$ \\
0.100 & -17.499 & -17.527 & \\
0.200 & -16.948 & -16.961 & \\
0.500 & -16.622 & -16.628 & $-v=w-$ \\
1.000 & -16.560 & -16.564 & - \\
\hline \hline
\end{tabular}




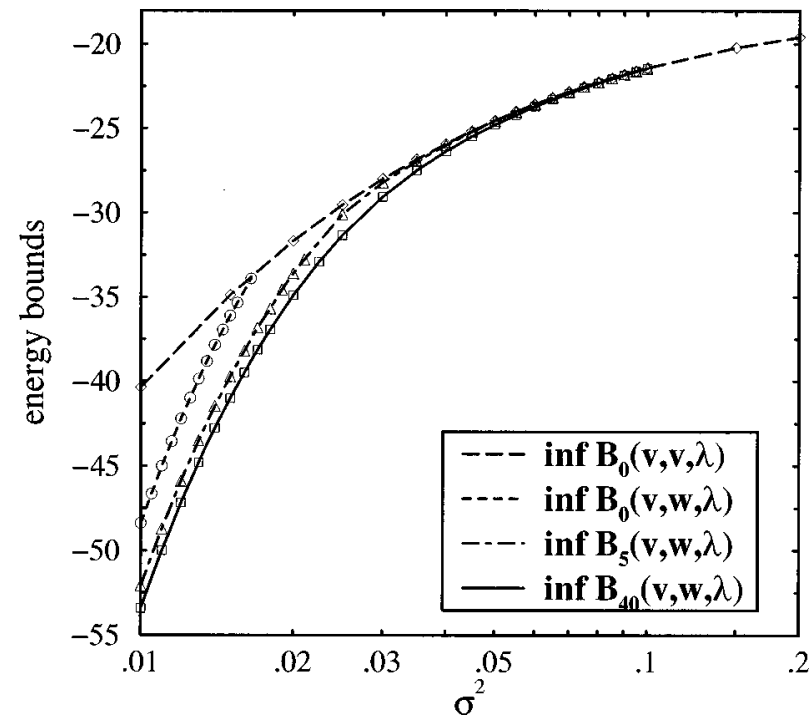

FIG. 1. Comparison of upper bounds on the ground-state energy as gained by minimization of the corresponding $B_{k}(v, w, \lambda) . \eta$ and $\xi$ are fixed as $\eta=1$ and $\xi=0.5$. The $\sigma$ dependence can directly be converted into an $\alpha_{M}$ dependence by means of Eq. (28).

phase-transition-like phenomena. As representative references, we quote Rashba ${ }^{10}$ and Sumi. ${ }^{11}$

Having in mind that $E_{0} / R_{0}$ was proven to be an analytical function of $\alpha_{M}$ (or, equivalently, $\sigma$ ) for all possible values of $\alpha_{M}$ (or, equivalently, $\sigma$ ), we evaluated $B_{k}$ for larger values of $k$. In Fig. 1, we show typical graphs of $\overline{B_{0}}, B_{0}, B_{5}$, and $B_{40}$ as functions of $\sigma^{2}$ ( $\eta^{2}$ and $\xi$ being fixed as indicated). The conclusion is again the same as in the case $D=3$ : For $k \rightarrow \infty$, no critical value of $\sigma^{2}$ exists. In so far, our numerical findings are consistent with the exact ones. ${ }^{12}$

In Table IV, we list bounds on $E_{0}$ for various materials of interest. In doing so, we are aware that a realistic theory for, e.g., GaAs should include at least the effects of the valenceband degeneracy as an obvious correction of the simple picture of a parabolic valence-band structure. Other corrections are due to spin-orbit coupling, exchange interaction, etc. Clearly, our theory does not include any of these. The most important application might be concerned with quantum-well structures. Unfortunately, the quantum-well problem exhibits additional problems, which are characterized by the key words "image charge" and "boundary conditions" (we refer to the paper by Altarelli ${ }^{14}$ ). Again, these are not included here. On the other hand, there seems to be a clear experimen-
TABLE IV. Absolute ground-state energy bounds $\overline{B_{0}}$ in $\mathrm{meV}$ as obtained in the present ansatz with material parameters listed in Table $\mathrm{V}$. Corresponding binding energies $\Delta E$, resulting from $\overline{B_{0}}$ by subtraction of the continuum edge $\Sigma$, in comparison with the results $\Delta E_{\mathrm{GI}}$ reported by Geddo and Iadonisi (Ref. 13).

\begin{tabular}{lcccc}
\hline \hline & $\overline{B_{0}}$ & $\Sigma$ & $\Delta E$ & $\Delta E_{G I}$ \\
\hline $\mathrm{GaAs}^{\mathrm{a}}$ & 20.71 & 8.29 & 12.44 & 12.45 \\
$\mathrm{GaAs}^{\mathrm{b}}$ & 34.04 & 14.25 & 19.79 & 19.89 \\
$\mathrm{CuCl}$ & 1471.6 & 490.7 & 980.9 & 1148.7 \\
$\mathrm{CdTe}$ & 102.18 & 44.10 & 58.08 & 59.68 \\
\hline \hline
\end{tabular}

${ }^{\text {a Light hole. }}$

${ }^{\mathrm{b}}$ Heavy hole.

tal evidence that two-dimensional features are observable (see, e.g., Oelgart et al. ${ }^{15}$ ) Our intention was to treat the exciton-phonon interaction as accurately as possible to have a well-defined basis for further improvements. For any ground-state energy bound we listed the corresponding continuum edge $\Sigma$, which is the reference for a calculation of the binding energy $\Delta E$, which can be found in a third column. $\Sigma$ is the sum of the polaronic self-energies of the electron and hole. It is important to use a precise value for $\Sigma$. In general, it is not sufficient to refer to the second-order perturbation result $-\hbar \omega\left(\alpha_{1}+\alpha_{2}\right)(\pi / 2)$ for $\Sigma$. One would systematically overestimate the binding energy that way, the mistake growing with $\alpha$. We give an example: for $\alpha=5$, the relative deviation of the self-energy from the second-order perturbation result is larger than $40 \%$. The lower the ground-state energy bound and $\Sigma$ are, the higher is the a priori reliability of the predicted value of the binding energy. The last column $\Delta E_{\mathrm{GI}}$ will be explained below.

As for the material parameters, we refer to Table V. These parameters are exactly those which were used by Geddo and Iadonisi. ${ }^{13}$ In fact, this paper is-to the best of our knowledge- the only one which can serve as a direct comparison. It presents a variational calculation of Lee-LowPines type, which is adequate for weak- or intermediatecoupling strength. Therefore, it is consistent that within this approach the continuum edge turns out to be $-\hbar \omega\left(\alpha_{1}\right.$ $\left.+\alpha_{2}\right)(\pi / 2)$. For large-coupling parameters (see, e.g., $\mathrm{CuCl}$ ) this result is grossly wrong, as indicated above. The authors do not present their primary results for the total energy, but only a series of binding energies $\Delta E_{\mathrm{GI}}$, which we list in Table IV. Our predictions and theirs differ particularly in the

TABLE V. Material parameters as employed to obtain the ground-state energy bounds $\overline{B_{0}}$ in Table IV. In the case of GaAs, Geddo and Iadonisi (Ref. 13) used different values for $\alpha_{h}$, namely, 0.096 and 0.173 instead of 0.075 and 0.177 . These are inconsistent with the well-established values of $\alpha_{e}$ and the masses as indicated. As the coupling is apparently small, the relative difference of the energies is small too.

\begin{tabular}{lccccccc}
\hline \hline & $\epsilon_{0}$ & $\epsilon_{\infty}$ & $m_{1}\left(m_{e}\right)$ & $m_{2}\left(m_{e}\right)$ & $\alpha_{e}$ & $\alpha_{h}$ & $\hbar \omega(\mathrm{meV})$ \\
\hline $\mathrm{GaAs}^{\mathrm{a}}$ & 13.1 & 11.1 & 0.0667 & 0.08 & 0.068 & $0.075^{*}$ & 36.8 \\
$\mathrm{GaAs}^{\mathrm{b}}$ & 13.1 & 11.1 & 0.0667 & 0.45 & 0.068 & $0.177^{*}$ & 36.8 \\
$\mathrm{CuCl}$ & 7.4 & 3.7 & 0.40 & 3.60 & 1.911 & 5.735 & 27.2 \\
$\mathrm{CdTe}$ & 10.3 & 6.9 & 0.088 & 0.60 & 0.364 & 0.950 & 20.7 \\
\hline \hline
\end{tabular}

${ }^{\mathrm{a}}$ Light hole.

${ }^{\mathrm{b}}$ Heavy hole. 
case of $\mathrm{CuCl}$. The discrepancy can be understood on the basis of our reasoning from above.

\section{CONCLUSIONS}

This paper is concerned with a qualitative and quantitative discussion of the ground-state energy of a two-dimensional exciton- (LO)-phonon system. We obtain an efficient upper bound, which gives correct results in the limits of a polaronic and a bare exciton, and treats the large-coupling case on the level of Feynman's polaron theory. The bound is a smooth function of the electron-phonon coupling parameter in the whole parameter domain. This is in agreement with rigorous qualitative results, and disproves previous assertions of a phase-transition-like behavior. We find as a by-product that the transition region between small and large coupling depends sensitively on the dimension. An accurate discussion of a two-dimensional system with $\alpha=5$ needs a theory which can provide an adequate treatment of the strongcoupling regime; a corresponding three-dimensional treatment is less critical.
Concerning the methodological part of this work, we use a variational procedure, based on a nonharmonic trial ansatz within the functional-integral approach. All interaction terms between electron and hole are modeled by a screened Coulomb potential, and the center-of-mass motion is described in analogy to Feynman's discussion of the free polaron. It turns out that all expressions can be reduced to integrals, involving the Coulomb Green function for $D=2$. To simplify these integrals further, it proves crucial to represent the Green function as a Fourier series.

\section{ACKNOWLEDGMENTS}

Financial support of the Deutsche Forschungsgemeinschaft is gratefully acknowledged. Parts of this work were performed within the Human Capital and Mobility Program of the European Commission. One of the authors (F.L.) acknowledges J. T. Devreese for financial support during the last stages of this work.
${ }^{1}$ H. Haken, Z. Phys. 147, 323 (1957).

${ }^{2}$ B. Gerlach and F. Luczak, Phys. Rev. B 54, 12841 (1997).

${ }^{3}$ The limiting cases are actually treated two times in Ref. 2. A qualitative discussion can be found in Sec. III, and a quantitative one in the Appendix. Unfortunately, the Appendix contains two misprints. The resolvent in Eqs. (A2) and (A3) has to be modified: In Eq. (A2) one should replace $R_{\infty}$ by $\left|\widetilde{E_{0}}\right|$ within the resolvent, and in Eq. (A3) one should replace $1+\widetilde{h}$ by $\left|\widetilde{E_{0}}\right| / R_{\infty}+\widetilde{h}$.

${ }^{4}$ L. C. Hostler, J. Math. Phys. 11, 2966 (1970); see also L. C. Hostler and R. H. Pratt, Phys. Rev. Lett. 10, 460 (1963). Hostler presented formulas (9) and (10) without proof. Instead, he referred to a preprint of other authors, which - to the best of our knowledge-was not published as a regular paper. The easiest way to verify the formulas of interest is to insert expansion (9) into the familiar differential equation for the Green function, and to solve this directly. In fact, this can be done in complete analogy to the three-dimensional treatment of Hostler and Pratt, which we quoted above.

${ }^{5}$ A. Erdelyi, W. Magnus, F. Oberhettinger, and F. G. Tricomi, Higher Transcendental Functions (McGraw-Hill, New York, 1953).
${ }^{6} \mathrm{H}$. Buchholz, Die Konfluente Hypergeometrische Function (Springer, Berlin, 1953), p. 86. English translation: The Confluent Hypergeometric Function (Springer, New York, 1969).

${ }^{7}$ A. Erdelyi, W. Magnus, F. Oberhettinger, and F. G. Tricomi, Tables of Integral Transforms (McGraw-Hill, New York, 1954). Here we specifically use formula (17) in Vol. 2.

${ }^{8}$ The formula of interest can be found, e.g., in Higher Transcendental Functions (Ref. 5), Vol. 2, p. 101, Eq. (31).

${ }^{9}$ W. Xiaoguang, F. M. Peeters, and J. T. Devreese, Phys. Rev. B 31, 3420 (1985).

${ }^{10}$ E. I. Rashba, Bull. Acad. Sci. USSR, Phys. Ser. 40, 20 (1976).

${ }^{11}$ A. Sumi, J. Phys. Soc. Jpn. 43, 1286 (1977).

${ }^{12}$ B. Gerlach and H. Löwen, Rev. Mod. Phys. 63, 63 (1991), and references therein.

${ }^{13}$ M. Geddo and G. Iadonisi, Nuovo Cimento D 12, 1641 (1990).

${ }^{14} \mathrm{M}$. Altarelli, in Heterojunctions and Semiconductor Superlattices, edited by G. Allan, G. Bastard, N. Boccara, M. Lannoo, and M. Voos, Proceedings of the Winter School Les Houches 1985 (Springer, Berlin, 1985).

${ }^{15}$ G. Oelgart, M. Proctor, D. Martin, F. Morier-Genaud, F.-K. Reinhart, B. Orschel, L. C. Andreani, and H. Rhan, Phys. Rev. 49, 10456 (1994). 